\title{
Effect of pyrolysis temperature on adsorption and degradation of THI and IMI by biochar
}

\author{
Siyao Wang, Hongbo Peng* \\ College of modern agricultural engineering, Kunming University of Science and Technology, Kunming 650500, China
}

\begin{abstract}
In order to explore the effects of biochar prepared at different pyrolysis temperatures and different solid-liquid ratios on the adsorption and degradation of thiacloprid and imidacloprid in biochar, three biochar were prepared by oxygen pyrolysis with sawdust and as raw materials at $300,500{ }^{\circ} \mathrm{C}$ and $600{ }^{\circ} \mathrm{C}$ respectively. The effects of biochar on the adsorption degradation of thiacloprid and Imidacloprid in biochar were studied. The results showed that the addition of biochar could significantly increase the soil $\mathrm{pH}$, available phosphorus and organic carbon content, and reduce the soil $\mathrm{H} / \mathrm{C}$. The addition of biochar significantly improved the adsorption of thiacloprid and imidacloprid, and the adsorption capacity increased with the increase of biochar pyrolysis temperature. Biochar with different pyrolysis temperatures had different effects on the degradation of thiacloprid and imidacloprid. The strong adsorption capacity of high temperature biochar system reduces the chemical degradation rate of thiacloprid and imidacloprid. Proper solid-liquid ratio can avoid the waste of adsorbent and achieve the optimal utilization rate. Therefore, the types and properties of biochar should be fully considered when using biochar to repair pesticide contaminated soil.
\end{abstract}

key word: Biochar; THI; IMI; adsorption; degradation

\section{Introduction}

Imidacloprid, also known as mimimidine, is a new nitromethylene insecticide. Its chemical name is 1-6chloro-3-pyridylmethyl-N-nitroimidazol-2-ylideneamine) Imidacloprid has a strong effect on insect nicotinic acetylesterase receptor, destroys the normal conduction of insect central nerve, and causes the rapid death of insect nerve paralysis. It has the characteristics of ultra-high efficiency and broad spectrum. The results of field efficacy test showed that imidacloprid had strong knockdown ability, rapid effect, significant control effect and long duration against black tailed leafhopper, planthopper (rice brown planthopper, gray Planthopper and white backed planthopper), aphid (cotton aphid and peach aphid) and thrips. It also had certain control effect on whitefly, rice borer, rice negative mud insect and rice weevil, and its performance was better than buprofezin, permethrin, Pirimicarb Fenitran and furbutan. In addition, imidacloprid has low toxicity to environmental organisms and low dosage, so it has a wide application prospect[14]. Imidacloprid contains nitroso, nitroguanidine, cyanamide and other pharmacokinetic groups. The neurotoxic substituent of imidacloprid is its nitroimine [57].

In recent years, pesticides have played an important role in controlling crop diseases and pests, but their extensive and inefficient use has led to serious soil pollution problems and toxic effects on most organisms, resulting in potential risks to agricultural soil and human health. The pharmacophores containing $=\mathrm{N}-\mathrm{NO}_{2},=\mathrm{NCN}$ and $=$ $\mathrm{C}(\mathrm{H}) \mathrm{NO}_{2}$ are important molecular characteristics of neonicotine pesticides. These highly electronegative pharmacophores can selectively identify the cationic binding sites on insect unique nicotinic acetylcholine receptors (nAChRs), but not with vertebrate nicotinic receptors $\alpha$ four $\beta 2$ subunit ( $\alpha$ four $\beta 2 \mathrm{nAChRs}$ )[8,9]. The main insecticidal mechanism is to act on the insect nervous system and its surrounding nerves to keep the insect excited, paralyze and die. Because there is no cross resistance between neonicotine insecticide and conventional insecticide, it has been widely used in crop planting. Only about $5 \%$ of its active components are absorbed by crops, and the unabsorbed parts enter into human body, soil and other media. The concentration detected in the environment is $\mu \mathrm{g} / \mathrm{L} \sim \mathrm{mg} / \mathrm{L}[10]$. In 2014, the global pesticide market sales were US $\$ 18.82$ billion, while the share of neonicotine pesticides exceeded $25 \%$. Neonicotine pesticide is frequently detected and widely used. Its impact on soil pollution and food safety has attracted extensive attention of researchers.

At present, a variety of technologies have been used to reduce the potential risk of soil pollution caused by pesticides, such as chemical redox and bioremediation, but these technologies have problems such as high cost and complex treatment [11]. On January 1, 2019, China

\footnotetext{
*Corresponding author: 673483508@qq.com
} 
began to implement the law of the people's Republic of China on the prevention and control of soil pollution, which puts forward higher requirements for soil pollution and remediation. Therefore, in order to reduce the absorption and transfer of pesticides in crops and ensure food safety, it is urgent to seek economic and effective remediation technologies for pesticide contaminated soil. Compost and biochar are common remediation agents for fixing or transforming pesticides [12], while biochar is considered as an environmental remediation agent for adsorbing and degrading pollutants to reduce the risk of soil pollution. It is obtained by pyrolysis of biomass under anoxic and low temperature $\left(<700{ }^{\circ} \mathrm{C}\right)$ [10]. Because biochar has large specific surface area and porous structure, it has strong adsorption capacity for heavy metals and pesticides in soil. The rich active oxygencontaining functional groups on the surface of biochar will transfer electrons and have high active oxygen generation ability, so as to promote its degradation of pesticides. Moreover, persistent free radicals on the surface of biochar or highly active small molecular free radicals induced by dissolved organic matter in biochar can directly oxidize and degrade p-nitrophenol, and these free radicals may directly degrade pesticides.

After biochar is applied to the soil, it will interact with dissolved organic matter (DOM), minerals and other components in the soil, block surface pores and reduce pesticide adsorption sites, thus affecting the adsorption of pesticides on biochar. It is found that the addition of biochar can improve the adsorption of imidacloprid, atrazine and isoproturon in soil system, However, due to the specific surface area and organic carbon of the mixed system of biochar and soil The actual value of organic carbon (OC) content is lower than the theoretical value, resulting in its actual adsorption capacity being lower than the theoretical adsorption capacity. Therefore, the adsorption capacity of biochar for pesticides in soil may be overestimated, which needs to be verified by more research data. Biochar remediation also has advantages and disadvantages for the microbial degradation of pesticides in soil. Previous studies have shown that biochar can improve the biodegradation of pesticides in soil. Soil microenvironment, improve soil microbial abundance, and then improve the degradation rate of pesticide microbial degradation. Firstly, due to the strong adsorption of biochar on pesticides, the utilization of pesticides by microorganisms and plants is reduced, which inhibits the microbial degradation of pesticides, and the inhibition increases with the increase of biochar dosage. Secondly, the degradable organic matter from the surface of biochar can promote the growth of microorganisms, but the strong adsorption of biochar reduces the utilization of such substances by microorganisms. The above differences in the effects of different biochar on microbial degradation of pesticides may be related to the pyrolysis temperature, raw materials, dosage and soil type of biochar, which needs further research.

\section{Material and method}

\subsection{Material}

The biomass wood sample used in this study was from Kunming University of science and technology. Technical-grade imidacloprid (IMI , 98.5\%) and thiacloprid (THI , 97\%) were purchased from the Aladdin Reagent Co., Ltd. (Shanghai, China). The basic properties of the two pesticides are listed in Table 1. Other chemicals and solvents were at least of analytical grade and used without further purification, except that the acetonitrile was chromatography pure. Ultra- pure (UP) water $(18.2 \mathrm{M} \Omega \cdot \mathrm{cm})$ was used to prepare all chemicals.

Table 1. Physicochemical properties of IMI and THI.

\begin{tabular}{|c|c|c|c|c|c|c|}
\hline $\begin{array}{c}\text { Compo } \\
\text { und }\end{array}$ & $\begin{array}{c}\text { Molecu } \\
\text { lar }\end{array}$ & $\begin{array}{c}\text { Mole } \\
\text { cular } \\
\text { weigh } \\
\text { t }\end{array}$ & $\begin{array}{l}\text { Water } \\
\text { solubility } \\
/ \mathrm{mg} \cdot \mathrm{L}^{-1}\end{array}$ & $\begin{array}{l}\lg \\
\mathrm{K}_{\mathrm{o}} \\
\mathrm{w}\end{array}$ & $\mathrm{pK}_{\mathrm{a}}$ & $\begin{array}{c}\text { Mole } \\
\text { cular } \\
\text { struct } \\
\text { ure }\end{array}$ \\
\hline $\begin{array}{c}\text { Imidacl } \\
\text { oprid }\end{array}$ & $\begin{array}{c}\mathrm{C}_{9} \mathrm{H}_{10} \mathrm{C} \\
1 \mathrm{~N}_{5} \mathrm{O}_{2}\end{array}$ & $\begin{array}{c}255.6 \\
6\end{array}$ & 510 & $\begin{array}{c}0.5 \\
7\end{array}$ & $\begin{array}{c}0.01 \pm \\
0.10\end{array}$ & \\
\hline $\begin{array}{c}\text { Thiaclo } \\
\text { prid }\end{array}$ & $\begin{array}{c}\mathrm{C}_{10} \mathrm{H}_{9} \mathrm{C} \\
1 \mathrm{~N}_{4} \mathrm{~S}\end{array}$ & $\begin{array}{c}252.7 \\
2 \\
\end{array}$ & 185 & 1.3 & $\begin{array}{c}0.01 \pm \\
0.10\end{array}$ & \\
\hline
\end{tabular}

Note: Kow data are taken from the PubChem datebase of Biotechnology Information(NCBI); Koc Organic carbon adsorption coefficient; $\mathrm{pKa}$ acidity coefficient.

\subsection{Preparation of biochar}

A lignin-rich biochar was used in this study. Wood was air-dried, ground, and passed through 100 mesh $(0.15 \mathrm{~mm})$ sieve. The dried feedstocks were charred at 300,500 and $600{ }^{\circ} \mathrm{C}$ for $4 \mathrm{~h}$ in a closed $300-\mathrm{mL}$ ceramic crucible with a cap under oxygen-limited conditions in a muffle furnace. The produced biochars were ground to pass through 100 mesh $(0.15 \mathrm{~mm})$ sieves and stored in $40-\mathrm{mL}$ glass jars.

\subsection{Adsorption experiments}

Batch adsorption equilibrium method was used in adsorption experiment. The specific steps were as follows : $80 \mathrm{mg}, 40 \mathrm{mg}, 8 \mathrm{mg}$ and $4 \mathrm{mg}$ biochar were weighed respectively in $4 \mathrm{~mL}$ EPA sample bottle (with a cover with Teflon gasket ), and then $4 \mathrm{~mL} 10 \mathrm{mg} / \mathrm{L} \mathrm{IMI}$ and THI solution containing $5 \mathrm{mmol} \cdot \mathrm{L}-1 \mathrm{NaCl}$ and $200 \mathrm{mg} \cdot \mathrm{L}-1$ $\mathrm{NaN} 3$ were added. Cover the cover tightly, shake well and place in $25 \pm 1{ }^{\circ} \mathrm{C}$ shaker to avoid light oscillation, oscillation speed $150 \mathrm{r} \cdot \mathrm{min}-1$. After oscillation for $144 \mathrm{~h}$, the sample bottle was taken out and centrifuged at 3500 $\mathrm{r} \cdot \mathrm{min}$-1 high-speed centrifuge for $10 \mathrm{~min}$. The supernatant was analyzed by HPLC after passing $0.45 \mu \mathrm{m}$ water filter membrane. Repeat 2 times per treatment.

\subsection{Degradation experiments}

This experiment only studied the chemical drop of IMI and THI. After $144 \mathrm{~h}$, the liquid in $4 \mathrm{~mL}$ flask was extracted completely, and $4 \mathrm{~mL}$ acetonitrile solution was added each time. After shaking well, it was placed in a 25 $\pm 1{ }^{\circ} \mathrm{C}$ shaker to avoid light oscillation, and the oscillation speed was $150 \mathrm{r} \cdot \mathrm{min}-1$. After oscillation for $3 \mathrm{~h}$, the sample bottle was taken out and centrifuged at 3500 $\mathrm{r} \cdot \mathrm{min}-1$ high-speed centrifuge for $10 \mathrm{~min}$. The supernatant was removed and determined by HPLC after passing 0.45 $\mu \mathrm{m}$ organic membrane. The extraction recoveries were 
above $90 \%$. The above solvents are pure chromatographic grade.

\section{Results and discussion}

\subsection{Effect of pyrolysis temperature on adsorption and degradation of THI and IMI by biochar}

The adsorption results of thiacloprid and imidacloprid in biochar are shown in Figure 1. As shown in Figure 1, it shows that the adsorption of thiamethoxam by biochar system is mainly multi-layer adsorption[23]. After adding biochar, the adsorption capacity of thiacloprid and imidacloprid was improved. The addition of biochar could significantly improve the adsorption capacity of soil for thiacloprid and imidacloprid, and the improvement degree of adsorption capacity increased with the increase of pyrolysis temperature of added biochar. The se of thiacloprid and imidacloprid in high temperature biochar system is higher than that in low temperature biochar system, which indicates that high temperature biochar has stronger adsorption capacity for thiacloprid and imidacloprid in soil. This is because during the hightemperature pyrolysis of sawdust, with the increase of pyrolysis temperature, the specific surface area of biochar increases, the degree of aromatization deepens and the surface hydrophobicity increases, which provides the possibility for biochar to adsorb pesticides[26]. Liu Jiannan et al. [27]also found that with the increase of pyrolysis temperature, the larger the specific surface area, the more adsorption sites can be provided on the surface of biochar, so the adsorption capacity of thiacloprid and imidacloprid on high-temperature biochar is strong. In addition, high temperature pyrolysis makes biochar contain more aromatic carbon and high specific surface area, so that thiacloprid and imidacloprid are adsorbed on biochar through hydrophobic distribution, pore filling, hydrogen bonding, $p(n)-\pi$ and $\pi-\pi$ electron donor acceptor interaction. The strong adsorption capacity in the high-temperature biochar system leads to the reduction of the concentration level of available nitrogen and soluble organic carbon. At the same time, this adsorption may compete with pollutant molecules for high-energy adsorption sites on the biochar surface or block micropores and mesopores[29], so as to reduce the adsorption sites of thiacloprid and imidacloprid. However, for the $300{ }^{\circ} \mathrm{C}$ biochar system, the release of available nitrogen, phosphorus and soluble organic carbon on the biochar surface changed its surface properties and improved the adsorption of thiacloprid and imidacloprid[31]. The adsorption of thiacloprid and imidacloprid by biochar still improved the adsorption capacity of soil due to biochar remediation, and the degree of improvement increased with the increase of biochar pyrolysis temperature. Therefore, the strong adsorption capacity of biochar to thiacloprid and imidacloprid can play an important role in reducing their mobility in soil.
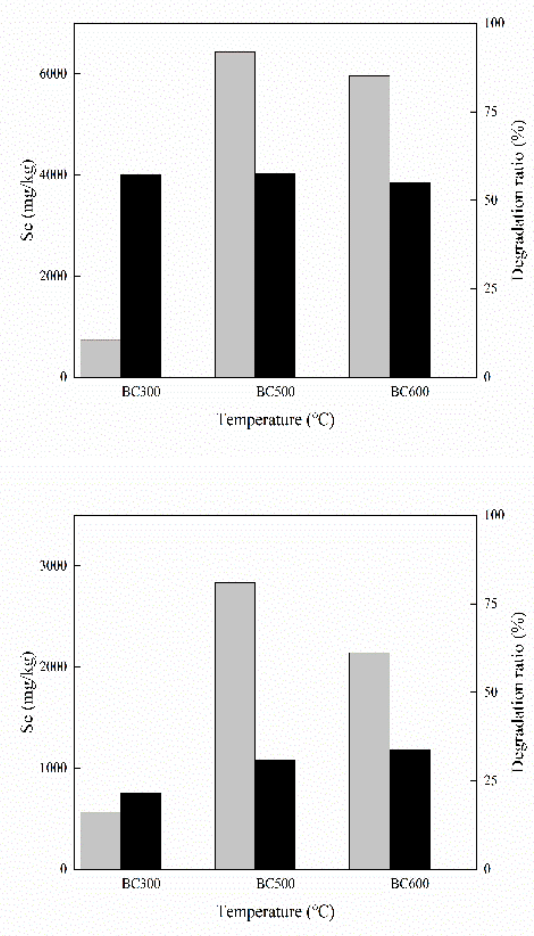

Figure 1. Adsorption and degradation of THI (left) and IMI (right) by biochar at different temperatures.

\subsection{Effect of solid-liquid ratio on adsorption and degradation of THI and IMI by biochar}

The experiment of solid-liquid ratio is mainly to explore the relationship between the input amount of adsorbent and the removal efficiency of thiacloprid and imidacloprid. If the input amount of adsorbent is too small, the amount of thiacloprid and imidacloprid can not be effectively removed. On the contrary, it will form a waste of resources and cause unnecessary economic losses. In order to fully improve the utilization of adsorbent, we did solid-liquid ratio experiment. The experimental results are shown in Figure 2、3 and 4.

As can be seen from Fig. 2、3 and 4, in the initial stage, the removal efficiency increases with the increase of adsorbent dosage. When the solid-liquid ratio reaches a certain value, even if the input of adsorbent increases, the adsorption capacity of thiacloprid and imidacloprid basically maintains a certain value without significant increase. When the solid-liquid ratio of biochar at $300{ }^{\circ} \mathrm{C}$ reaches 1:100, the removal efficiency is almost the highest, and there is no increase. According to the highest removal efficiency and cost-effectiveness, the best solidliquid ratio is $1: 100$. At the same time, the best solidliquid ratio of biochar at $500{ }^{\circ} \mathrm{C}$ and $600{ }^{\circ} \mathrm{C}$ is $1: 1000$. 

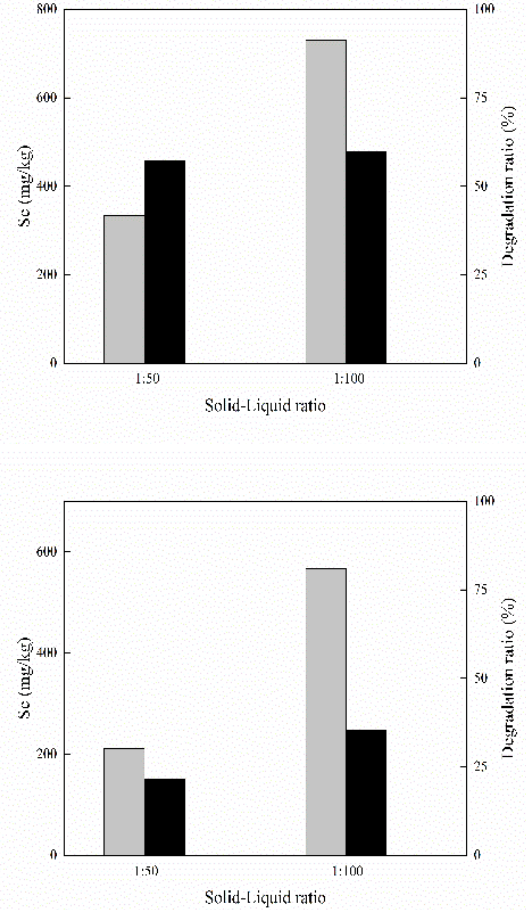

Figure 2. Adsorption and degradation of thiacloprid (left) and imidacloprid (right) by biochar at $300{ }^{\circ} \mathrm{C}$ under different solidliquid ratio
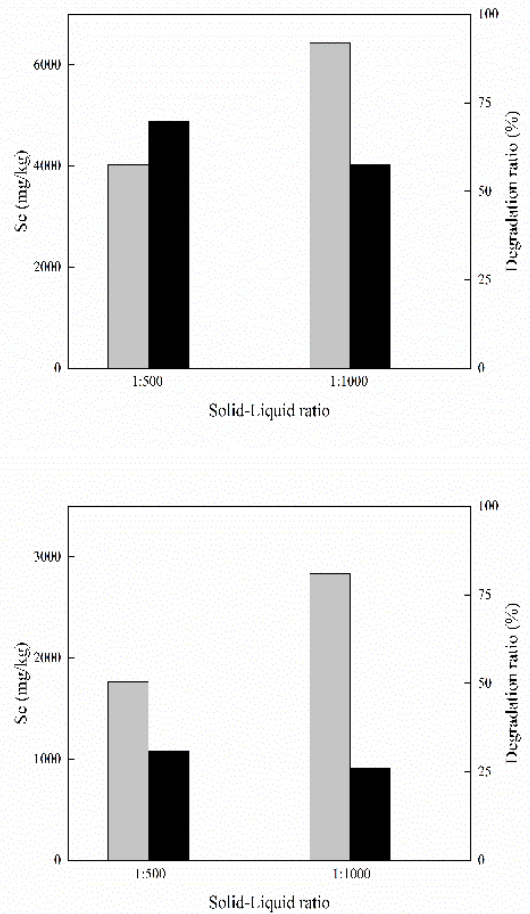

Figure 3. Adsorption and degradation of thiacloprid (left) and imidacloprid (right) by biochar at $500{ }^{\circ} \mathrm{C}$ under different solidliquid ratio
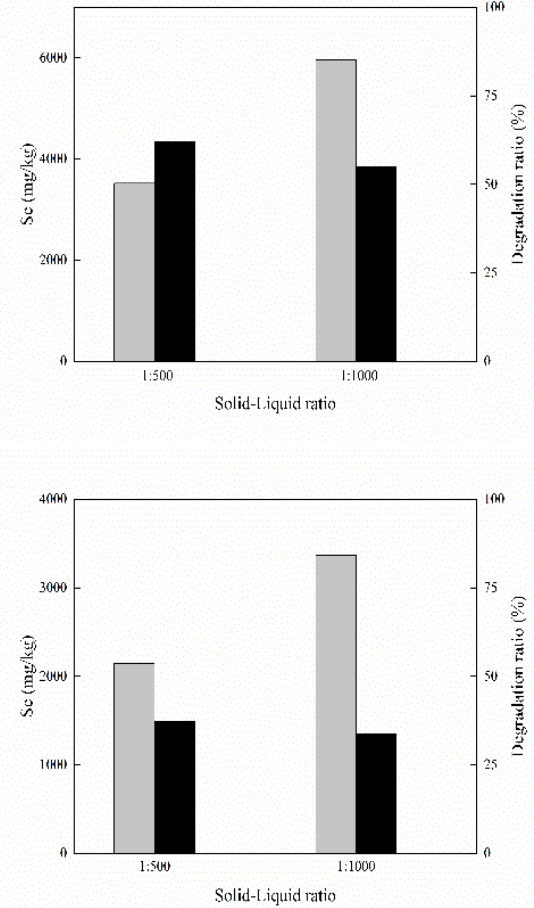

Figure 4. Adsorption and degradation of thiacloprid (left) and imidacloprid (right) by biochar at $600{ }^{\circ} \mathrm{C}$ under different solidliquid ratio

\subsection{Adsorption and degradation mechanism of THI and IMI under biochar}

Other studies have pointed out that for the adsorption of thiacloprid and imidacloprid by the same biochar, the adsorption of biochar at $500^{\circ} \mathrm{C}$ is higher than that of biochar at $300^{\circ} \mathrm{C}$. Compared with $300^{\circ} \mathrm{C}$ biochar, $500^{\circ}$ $\mathrm{C}$ biochar has relatively fewer polar functional groups, higher aromaticity and stronger hydrophobicity. Therefore, it is speculated that hydrophobicity plays a dominant role in the adsorption of thiacloprid and imidacloprid on biochar, and there may also be adsorption mechanisms such as pore filling, $\pi-\pi$ electron donor receptor and hydrogen bond, which affect the adsorption of thiacloprid and imidacloprid. Some studies have also shown that the adsorption of thiacloprid and imidacloprid on biochar will increase with the increase of organic carbon content; The study also pointed out that when the complex adsorbs hydrophobic organic pollutants, hydrophobicity is the dominant mechanism controlling its adsorption. In conclusion, the adsorption mechanism of thiacloprid and imidacloprid on biochar is mainly hydrophobic and $\pi-\pi$ effect.

The - OH peak at $3420 \mathrm{~cm}-1$ and the aromatic $-\mathrm{C}=\mathrm{C}$ peak at $1610 \mathrm{~cm}-1$ of sawdust biochar decreased significantly with the decrease of temperature (Fig. 5), indicating the infrared characteristics of thiacloprid and imidacloprid before and after adsorption on biochar at 300 ${ }^{\circ} \mathrm{C}, 400{ }^{\circ} \mathrm{C}$ and $500{ }^{\circ} \mathrm{C}$. It was found that the $-\mathrm{OH}$ peak at $3420 \mathrm{~cm}-1$ of thiacloprid and imidacloprid adsorbed complex was not different from the original complex, However, the aromatic- $\mathrm{C}=\mathrm{C}$ peak of the complex adsorbed by thiacloprid and imidacloprid at $1610 \mathrm{~cm}-1$ is 
much lower than that before the corresponding reaction. The - $\mathrm{COOH}$ of biochar may form a $\pi$ electron conjugated system with its aromatic ring. Through $p / \pi-\pi$ interaction with the benzene ring of thiacloprid and imidacloprid, phenanthrene interacts strongly with the complex. In addition, thiacloprid and imidacloprid can be directly combined with the aromatic components of biochar through $\pi-\pi$ interaction. Of course, the contribution of aliphatic components to the adsorption of thiacloprid and imidacloprid can not be ignored. In conclusion, the adsorption mechanisms of thiacloprid and imidacloprid on biochar are mainly hydrophobic, $\mathrm{p} / \pi-\pi$ and $\pi-\pi$.
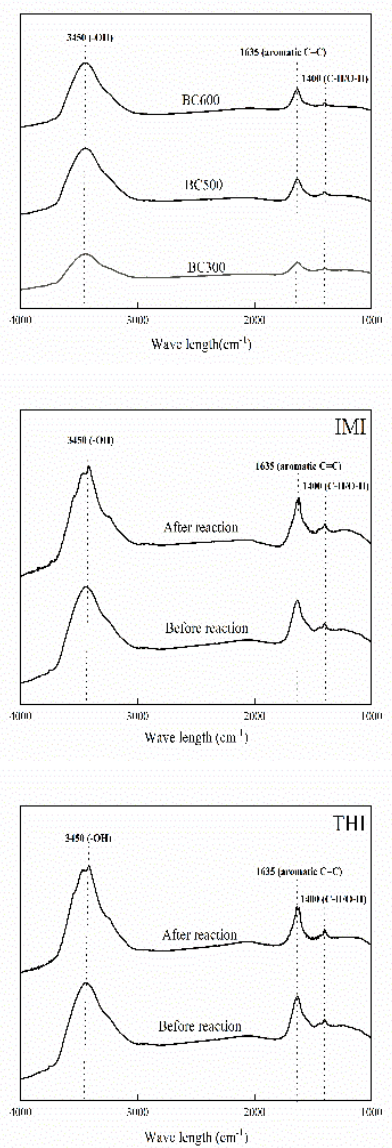

Figure 5. FT-IR

\section{Conclusion}

(1) Adsorption of thiacloprid and imidacloprid on biochar system biochar at different pyrolysis temperatures affects the adsorption and degradation of thiacloprid and imidacloprid by affecting the physical and chemical properties of biochar.

(2) Thiamethoxam has relatively high chemical degradation rate in high temperature biochar system, but relatively low in low temperature biochar system. Biochar enhanced the adsorption and degradation of thiacloprid and imidacloprid by increasing the content of organic carbon.

(3) At the same temperature, large solid-liquid ratio does not necessarily have strong adsorption and degradation ability. Biochar has a certain adsorption capacity and will not continue to adsorb after reaching saturation. Therefore, it is very important to find an appropriate solid-liquid ratio.

\section{References}

1. Chen, LG Determination of imidacloprid in rice by molecularly imprinted-matrix solid-phase dispersion with liquid chromatography tandem mass spectrometry[J]. J CHROMATOGR B, 2012, 2012,897(-): 32-36.

2. Pandiselvi S, Sathiyanarayanan S, Ramesh A Determination of Spirotetramat and Imidacloprid Residues in Cotton Seed, Lint, Oil and Soil by HPLC UV Method and their Dissipation in Cotton Plant[J]. Pesticide Research Journal, 2010, 22: 168-173.

3. Iqbal, S., Uddin, et al. Extraction, Cleanup, and Chromatographic Determination of Imidacloprid Residues in Wheat[J]. BULLETIN OF ENVIRONMENTAL CONTAMINATION AND TOXICOLOGY, 2012:

4. Li Y, Yang L, Yan H, et al. Uptake, translocation and accumulation of imidacloprid in six leafy vegetables at three growth stages[J]. Ecotoxicology and Environmental Safety, 2018, 164: 690-695.

5. Michlig M P, Julieta M, Pacini A C, et al. Determination of imidacloprid in beehive samples by UHPLC-MS/MS[J]. Microchemical Journal, 2018, 143: S0026265X18303278-.

6. Wang S W, Zeng G F, Liu Y P, et al. Determination of Thiacloprid,Spirotetramat and Its Metabolites Residues in Litchi and Longan by High Performance Liquid Chromatography-Tandem Mass Spectrometry[J]. Journal of Instrumental Analysis, 2019:

7. Min, Huang, Xinxian, et al. A liquid chromatography with tandem mass spectrometry method to simultaneously determinate chlorpyrifos, imidacloprid and imidacloprid metabolites in wheat[J]. Journal of separation science, 2019:

8. Angioni A, Porcu L, Pirisi F LC/DAD/ESI/MS method for the determination of imidacloprid, thiacloprid, and spinosad in olives and olive oil after field treatment[J]. Journal of Agricultural \& Food Chemistry, 2011, 59(20): 11359-11366.

9. Auteri D, Arena M, Barmaz S, et al. Neonicotinoids and bees: The case of the European regulatory risk assessment - ScienceDirect[J]. Science of the Total Environment, 2017, 579(feb.1): 966-971.

10. Mommaerts V, Reynders S, Boulet J, et al. Risk assessment for side-effects of neonicotinoids against bumblebees with and without impairing foraging behavior[J]. Ecotoxicology, 2010, 19(1): 207-215.

11. Wu B, Cheng G, Kai J, et al. Mycoextraction by Clitocybe maxima combined with metal immobilization by biochar and activated carbon in an aged soil[J]. Science of the Total Environment, 2016, 562(aug.15): 732-739. 
12. Richard, S., Quilliam, et al. Life in the 'charosphere' - Does biochar in agricultural soil provide a significant habitat for microorganisms?[J]. Soil Biology and Biochemistry, 2013, 65(1): 287-293.

13. Deng J H, Gao L Y, Zhou W W, et al. Adsorption characteristics and mechanisms of $\mathrm{Cd} 2+$ in biochar derived fromvetiver grass under different pyrolysis temperatures[J]. Journal of Agro-Environment Science, 2018:

14. Charisma, Lattao, Xiaoyan, et al. Influence of Molecular Structure and Adsorbent Properties on Sorption of Organic Compounds to a Temperature Series of Wood Chars[J]. Environmental Science \& Technology: ES\&T, 2014, 48(9): 4790-4798.

15. Liu J N, Feng J M, Dan L I, et al. The adsorption of $\mathrm{Cd} \sim(2+)$ and $\mathrm{Zn} \sim(2+)$ in aqueous solutions by dairy manure and walnut shell biochar[J]. Journal of AgroEnvironment Science, 2019:

16. Ren G, Teng Y, Ren W, et al. Pyrene dissipation potential varies with soil type and associated bacterial community changes[J]. Soil Biology and Biochemistry, 2016:

17. Ke, Sun, Mingjie, et al. Impact of Deashing Treatment on Biochar Structural Properties and Potential Sorption Mechanisms of Phenanthrene[J]. Environmental Science \& Technology, 2013: 